\title{
Techniques for Preservation of the Frontotemporal Branch of Facial Nerve during Orbitozygomatic Approaches
}

\author{
Toma Spiriev ${ }^{1}$ Lars Poulsgaard $^{1}$ Kaare Fugleholm ${ }^{1}$ \\ ${ }^{1}$ Department of Neurosurgery, Rigshospitalet-Copenhagen University \\ Hospital, Copenhagen, Denmark \\ Address for correspondence Toma Spiriev, MD, Department of \\ Neurosurgery, Rigshospitalet-Copenhagen University Hospital, \\ Blegdamsvej 9, 2100 Copenhagen Ø, Copenhagen, Denmark \\ J Neurol Surg B 2015;76:189-194. \\ (e-mail: spiriev@gmail.com).
}

\begin{abstract}
Background During orbitozygomatic (OZ) approaches, the frontotemporal branch (FTB) of the facial nerve is exposed to injury if proper measures are not taken. This article describes in detail the nuances of the two most common techniques (interfascial and subfascial dissection).

Design The FTB of the facial nerve was dissected and followed in its tissue planes on fresh-frozen cadaver heads. The interfascial and subfascial dissections were performed, and every step was photographed and examined.

Results The interfascial dissection is safe to be started from the most anterior part of the superior temporal line and followed to the root of the zygoma. The dissection is continued on the deep temporalis fascia (DTF), and the interfascial fat pad is elevated. With the subfascial dissection, both the superficial temporalis fascia and the DTF are

Keywords

- facial nerve

- orbitozygomatic craniotomy

- operative technique

- skull base surgery elevated. The interfascial dissection exposes the zygomatic arch directly, whereas the subfascial dissection requires an additional cut on the DTF to expose the zygomatic arch. Proper subperiosteal dissection on the zygomatic arch is another important step in FTB preservation.

Conclusion Detailed understanding of the complex relationship of the tissue planes in the frontotemporal region is needed to perform $\mathrm{OZ}$ exposures safely.
\end{abstract}

\section{Introduction}

The orbitozygomatic (OZ) approach is a common choice for difficult lesions in the parasellar and perimesencephalic area, cavernous sinus, and petroclival region. ${ }^{1-10}$ However, the extensive soft tissue dissection necessary to expose the zygomatic arch and lateral orbital rim poses a risk to the frontotemporal branch (FTB) of the facial nerve. Damage to the FTB results in an unpleasant cosmetic defect in the face caused by weakness of the orbicularis oculi, frontalis, and corrugator supercilii muscles. ${ }^{11-16}$ Therefore preservation of the FTB of the facial nerve requires a detailed understanding of its course and the complex fascial planes in the frontotemporal region.

received

April 6, 2014

accepted after revision

September 16, 2014

published online

December 24, 2014

Two main techniques for FTB preservation during OZ craniotomy are described in the literature: the interfascial ${ }^{16}$ and the subfascial ${ }^{11,14}$ dissections. The present study offers a detailed description of the technical nuances of both techniques and of the relevant anatomy of the frontotemporal region.

\section{Material and Methods}

Four frontotemporal regions (two cadaveric heads) were studied. The specimens were fresh-frozen thawed, which allows for good preservation of individual tissue planes and precise identification of the neurovascular structures in the

(c) 2015 Georg Thieme Verlag KG Stuttgart · New York
DOI http://dx.doi.org/ 10.1055/s-0034-1396599. ISSN 2193-6331. 
anatomical regions of interest. The facial nerve was identified at its exit from the stylomastoid foramen and dissected distally; the FTB of the facial nerve was carefully dissected and followed in its tissue planes. The interfascial and subfascial dissections were performed, and every step was photographed and examined. A review of the literature was carried out.

\section{Results}

\section{Interfascial Dissection}

The skin incision begins just in front of the tragus, extends superiorly to the superotemporal crest, then curves anteriorly, crossing the midline. The incision is maintained behind the hairline for the entire course (-Fig. 1). A larger skin incision allows less retraction of the flap and avoids retractioninduced injury to the FTB. ${ }^{8,10}$ The galea is separated from the pericranium in the loose areolar tissue plane (between the pericranium and galea) above the superior temporal line, and inferiorly the temporoparietal fascia (TPF) is then separated from the temporalis fascia in the same loose areolar tissue plane. Care is taken not to extend the dissection too inferiorly (below the level of the frontal process of zygoma) in the anterior zygomaticotemporal region because the loose areolar tissue between the TPF and the temporalis fascia is absent, and there is loss of the dissection plane and a greater risk of injury to the FTB of the facial nerve in this region (-Fig. 1). ${ }^{15,17}$ Once the landmark where these adhesions start is reached (inferior temporal septum), it is safer to keep the dissection plane in the loose areolar tissue between the TPF and temporalis fascia close to the superior temporal line. In the superolateral orbital rim area, the loose areolar tissue between the TPF and the superficial temporalis fascia (STF) is present and easily dissectible. After the skin flap is raised, the interfascial splitting is started from the most anterior part of the superior temporal line where the STF and the deep temporalis fascia (DTF) are continuous and easily identifiable. The STF is cut with scissors, and dissection is continued obliquely toward the root of the zygoma (the beginning of the skin incision in front of the tragus). The dissection is continued on the DTF, below the interfascial fat pad, and the fat pad is elevated together with the STF thus exposing the lateral orbital rim. The final key step is subperiosteal dissection on the zygoma and lateral orbital rim that reveals the orbitozygomatic bar.

\section{Subfascial Dissection}

The same skin incision and initial steps are performed until the temporalis fascia is exposed at the beginning of the incision (-Fig. 2). The temporalis fascia is incised along the skin incision to the temporalis muscle fibers, and the dissection is started between the muscle fibers and the temporalis fascia. Another temporalis fascia cut is made along the superior temporal line (STL). On the STL a fascial cuff is left that is used for later closure (-Fig. 2). The dissection is continued along the superior temporal line anteriorly to the lateral orbital rim. The temporalis fascia splits into superficial and deep layers $\sim 2 \mathrm{~cm}$ before its insertion on
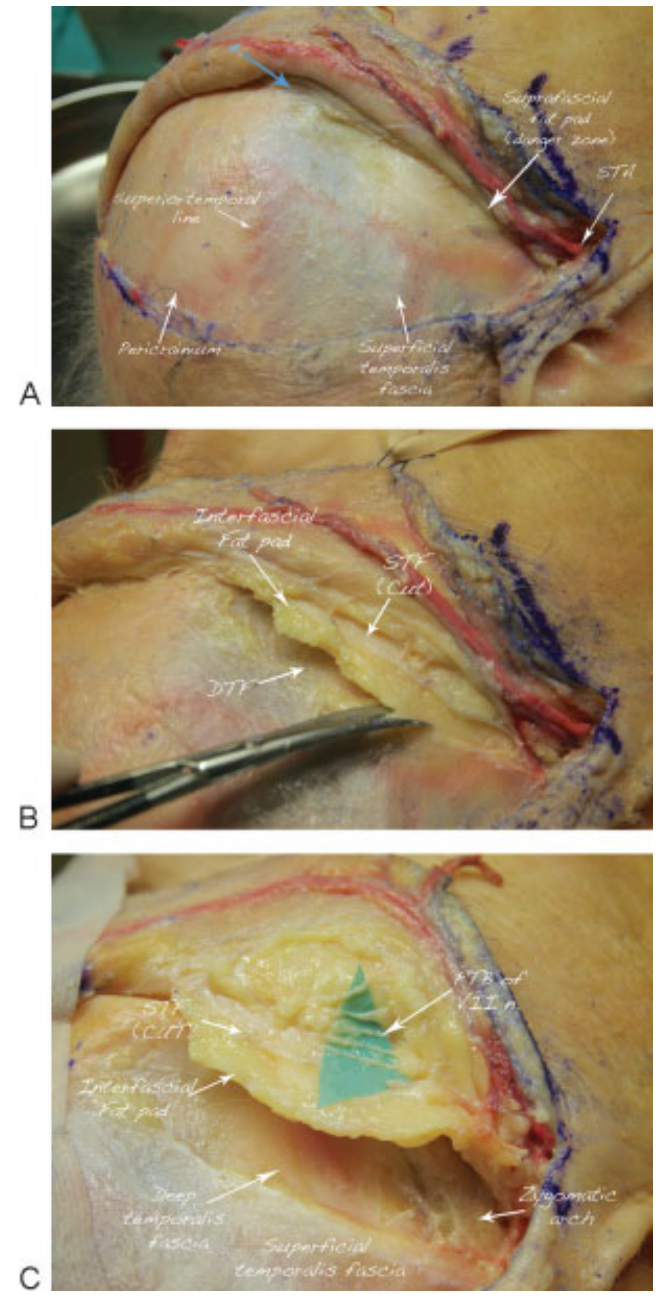

Fig. 1 Interfascial dissection presented in a stepwise fashion: right frontotemporal region. (A) Skin incision used for the right orbitozygomatic approach. The incision starts at the zygomatic arch, just in front of the tragus, continues to the superior temporal line, curves just behind the hairline, and reaches the midline. Dissection of the superficial tissue planes at the beginning of the skin incision: The skin and the subcutaneous fat are separated from the temporoparietal fascia (TPF), and the TPF is dissected from the superficial temporalis fascia (STF) in the loose areolar tissue plane, located between these two fascial layers. The superficial temporal artery (STA) most commonly runs within the TPF and is dissected and protected. The blue arrow points to the beginning of the interfascial dissection on the anterior part of the superior temporal line where the STF and the deep temporalis fascia (DTF) are continuous. In the inferior and anterior portion of the dissection, in the zygomaticotemporal angle, the suprafascial fat pad is seen. This is the danger zone for the FTB of the facial nerve because of the thick adhesions between the TPF and STF. In contrast to the superolateral orbital angle, where the loose areolar tissue between the TPF and the STF is very well developed, in the inferior zygomaticotemporal angle there is no loose areolar tissue in this area, respectively, and no dissection plane between the TPF and the STF. The FTB of facial nerve is exposed to injury if dissection is continued in this area. (B) The STF is cut. The DTF and the interfascial fat pad are identified. The STF cut is extended obliquely toward the root of the zygoma, thus avoiding the anterior zygomaticotemporal angle. By lifting the STF with the tip of the scissors before each cut, there is no danger of slipping between the individual tissue planes. After the STF is cut, the interfascial fad pad is elevated, and the dissection is kept close to the DTF toward the lateral orbital rim. As long as the dissection is below the interfascial fat pad on the DTF, there is little danger for the FTB of the facial nerve. (C) Microsurgical dissection of the branches of the FTB of the facial nerve after the interfascial dissection was performed. Note the localization of the branches above the superficial leaflet of the temporalis fascia. 

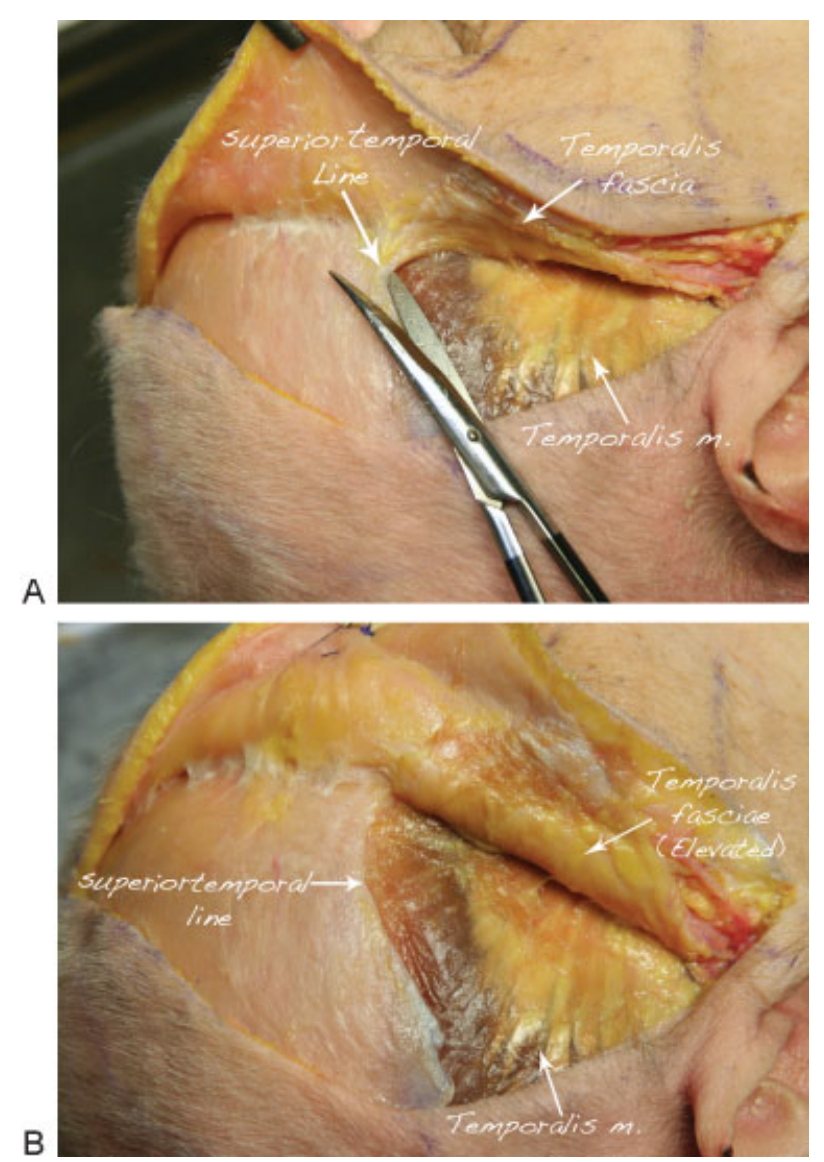

Fig. 2 Subfascial dissection presented in a stepwise fashion: skin incision for the right orbitozygomatic approach as described in - Fig. 1. (A) The subfascial dissection begins by identifying the temporalis fascia at the beginning of the skin incision. The temporalis fascia is incised to the muscle fibers, and the dissection is continued between the muscle and the temporalis fascia. The temporalis fascia splits into superficial and deep layers $\sim 2 \mathrm{~cm}$ before its insertion on the lateral orbital rim and zygomatic arch. (B) By keeping the dissection in the tissue plane between the muscle fiber and the temporalis fascia, both fascial layers (superficial and deep temporalis fasciae) are elevated together with the interfascial fat pad. On the superior temporal line, a fascial cuff is left that is used for later closure.

the lateral orbital rim and zygomatic arch. By keeping the dissection in the tissue plane between the muscle fiber and the temporalis fascia, both fascial layers (superficial and DTF) are elevated together with the interfascial fat pad. The main difference compared with the interfascial technique is that an additional cut on the DTF should be made to expose the bone at the lateral orbital rim and on the zygomatic arch (-Fig. 3 ). The final key step is subperiosteal dissection on the zygomatic arch and lateral orbital rim that reveals the orbitozygomatic bar. By performing the subfascial dissection, both STF and DTF are elevated together with the fat pad providing a wide exposure of zygomatic bone and lateral orbital rim ( - Fig. 4).

\section{Discussion}

The FTB is at risk of injury during soft tissue dissection using $\mathrm{OZ}$ approaches. If the proper technique for FTB preservation is not used, the incidence of FTB palsy could
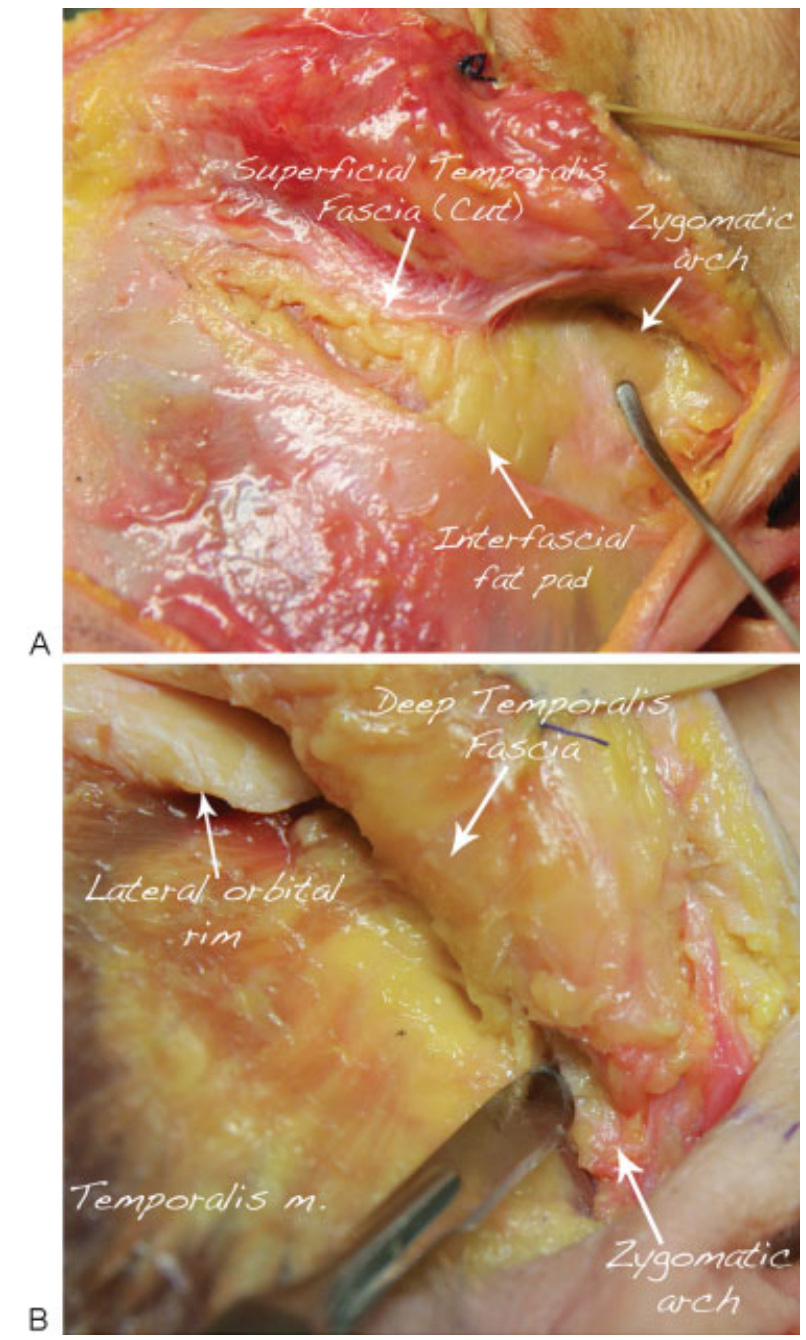

Fig. 3 Differences between the interfascial and subfascial dissections. (A) With the interfascial dissection, the root of the zygoma is exposed directly, where subperiosteal dissection should be continued. (B) With the subfascial dissection, an additional cut on the deep temporalis fascia should be done on the zygomatic arch and lateral orbital rim to expose the bone where a subperiosteal dissection should be continued.

reach $30 \% .^{13,15}$ Therefore to perform these approaches correctly they should be studied in the context of the relevant anatomy of the complex course of the FTB of the facial nerve and the individual fascial planes in the frontotemporal region.

\section{The Frontotemporal Branch of Facial Nerve Anatomy}

The VII nerve exits the foramen stylomastoideum, crosses the mandible $\sim 2.5 \mathrm{~cm}$ below the zygomatic arch, and enters the parotid gland where it divides into five branches: frontal, zygomatic, buccal, mandibular, and cervical (- Figs. 1 and 5). The FTB branch of the facial nerve crosses the zygomatic arch $\sim 2.0 \mathrm{~cm}$ (range: $0.8-3.1 \mathrm{~cm}$ ) from the tragus and gives three main rami: the auricularis (posterior), frontalis (middle), and orbicularis (anterior). ${ }^{14-16,18-20}$ At the level of the zygomatic arch, the FTB branches cross in the fibro-fatty layer underneath the TPF. 15,17,19,21,22 The ramus auricularis runs posteriorly and innervates the temporoparietal, auricular, and 

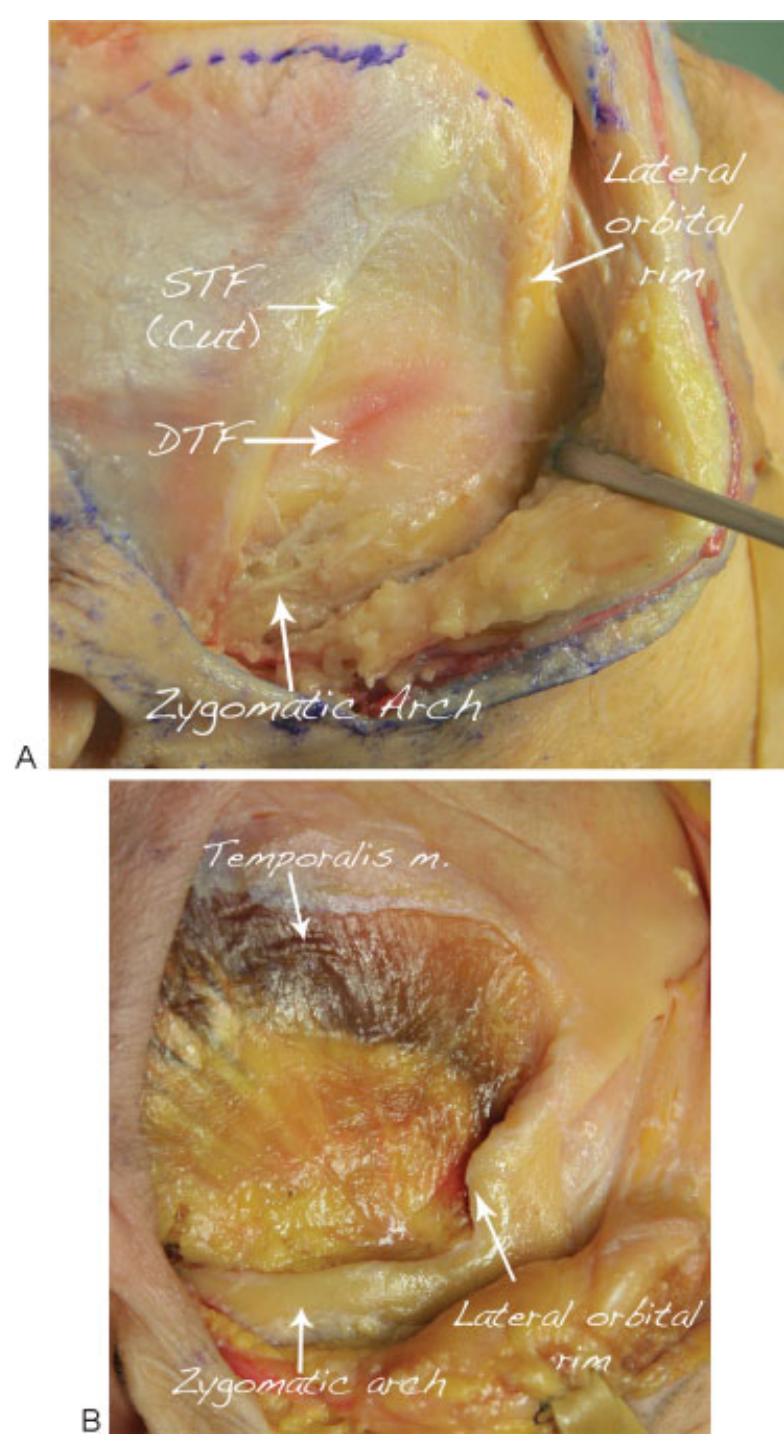

Fig. 4 Final view after right-sided (A) interfascial and (B) subfascial dissections. Both techniques allow for appropriate zygoma and lateral orbital rim exposures needed for the orbitozygomatic approaches. DTF, deep temporalis fascia; STF, superficial temporalis fascia.

tragal muscles. ${ }^{14-16,18-20}$ Superior to the zygomatic arch, the FTB branches run within the TPF. 11,12,15,18,19,22-24

The ramus frontalis (middle ramus) running within the TPF innervates the musculus frontalis. ${ }^{12,13,15,17,19,21,23}$ This was also confirmed in our study. The location of the frontal (middle) ramus, which is the longest one, above the zygomatic arch is located just above the TPF (we divided the TPF to expose the nerve). Several small twigs, ramus orbicularis or anterior ramus, give innervation to the musculus orbicularis oculi. ${ }^{11,12,14,18,19,22,25}$ These branches runs in the fat pad above the superficial leaflet of the temporalis fascia, in an area known as the inferior temporal septum, where the TPF adherens to the superficial layer of the DTF. ${ }^{15,17,19}$ After cutting through this septum or adhesion, one can find the FTBs of the facial nerve behind it in a fibro-fatty layer ${ }^{15,17,19}$ (-Fig. 1).

According to Ammirati et al, ${ }^{11,12}$ occasionally a twig for the frontalis muscle may run in the fat pad between the two

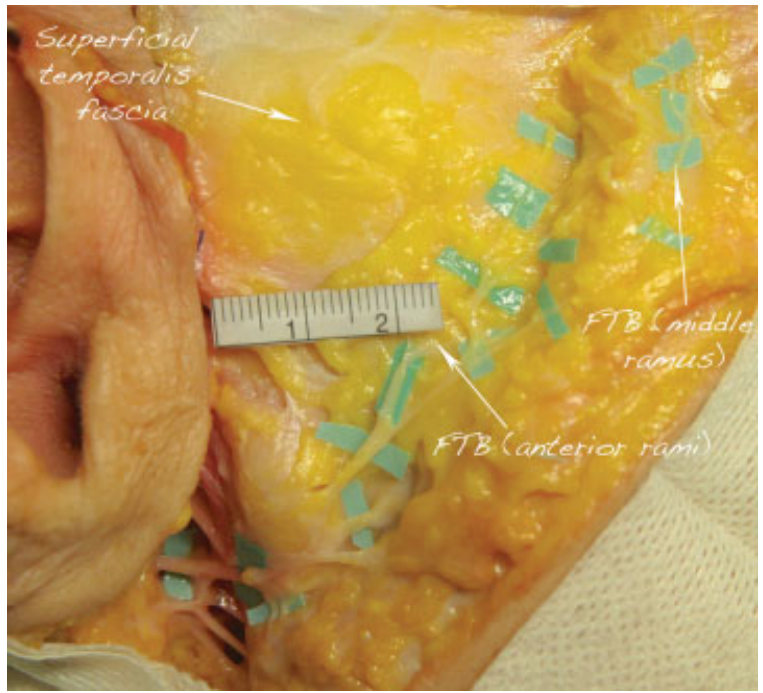

Fig. 5 Microsurgical dissection of the frontotemporal (FTB) of the facial nerve (middle and anterior rami). The VII nerve exits the foramen stylomastoideum, crosses the mandible $\sim 2.5 \mathrm{~cm}$ below the zygomatic arch, and enters the parotid gland where it divides into five branches: frontal, zygomatic, buccal, mandibular, and cervical. The FTB branch of the facial nerve crosses the zygomatic arch $\sim 2.0 \mathrm{~cm}$ (range: $0.8-3.1$ $\mathrm{cm}$ ) from the tragus and divides into three branches: the auricularis (not presented), frontalis (middle), and orbicularis (anterior). The ramus frontalis (middle ramus) runs just above the temporoparietal fascia (TPF) and innervates the frontalis muscle. Several small twigs (ramus orbicularis or anterior ramus) run in the fat pad above the superficial leaflet of the temporalis fascia innervating the orbicularis oculi muscle. Branches from the zygomatic nerve innervate the latter also. Anteriorly the superficial temporalis fascia (STF) is very thin, and the interfascial (between the STF and deep temporalis fascia) fat pad could be visualized.

layers of the superficial temporal fascia, and therefore the Yasargil interfascial dissection may at times not protect the FTB. However, this was not confirmed in other studies, and some authors argue that these aberrant twigs could actually be branches of the zygomaticotemporal nerve. ${ }^{19}$ This nerve is a branch of the zygomatic nerve that emerges from the zygomaticotemporal foramen, pierces the DTF adjacent to the zygomaticofrontal suture, and divides into multiple branches running parallel to perforating vessels originating from the middle temporal artery. Then these branches cross the superficial temporal fascia, cross the fibro-fatty tissue, and pierce the TPF to supply the skin over the temporal region. ${ }^{17,19,26}$

During our dissections we did not find such an aberrant twig of the FTB, but this might be due to the limited number of specimens dissected (two in our study) to detect such aberrant twigs of the FTB.

In their article, Coscarella et $\mathrm{al}^{14}$ point out that the superficial temporal artery (STA) could serve as a potential landmark to help guide subfascial or interfascial dissection during $\mathrm{OZ}$ approaches. In their study the ramus frontalis appeared to run parallel and just caudal to the frontal branch of the STA. There is general agreement that the FTB of the facial nerve is anteroinferior to the frontal branch of the STA. ${ }^{18,19}$ However, Lei et $\mathrm{al}^{20}$ found that this relationship 
varied according to the point of the STA bifurcation. If the STA bifurcates above the horizontal line of the superior orbital rim, the frontal branch is consistently anteroinferior to the STA. However, if STA bifurcation occurred below this line, one or more branches of the nerve could be located superior to, or interweaving with, the STA.

\section{Individual Tissue Planes in the Temporal Region and Their Relevance to the Technique for FTB Preservation} The FTB of the facial nerve runs in a complex relation to the musculoaponeurotic system of the frontotemporal region. The TPF above the superior temporal line is a single layer continuous with the galea, and below the zygomatic arch it is continuous with the musculoaponeurotic system of the face. ${ }^{11,18,19}$ Between the TPF and temporal fascia is a layer of loose areolar tissue that is very well present superiorly over the superior temporal line and lost along a curved line between the superolateral border of the orbit and the zygomatic arch. ${ }^{15,17,19}$

The temporal fascia is a single layer over the upper and posterior part of the temporalis muscle. However, 1 to $2 \mathrm{~cm}$ before its insertion on the lateral orbital rim and zygomatic arch, the temporal fascia splits into two layers, the STF and the DTF, that attach anteriorly to the lateral and medial borders of the zygomatic arch. ${ }^{11,15,16,18,19}$ Between these two layers is a constant sickle-shaped interfascial fat pad. ${ }^{16}$ However, two other distinct fat pads are described in the frontotemporal region: the suprafascial fat pad that lies anteriorly in the zygomaticotemporal region in a plane between the TPF and STF and the subfascial fat pad that overlies the temporal muscle and is located below the DTF. ${ }^{11,18}$

As Salas et al report ${ }^{15}$ in their detailed anatomical and histologic study of the frontotemporal region and the FTB of the facial nerve, above the zygomatic arch the nerve runs in the deepest part of the TPF. In the most anterior zygomaticotemporal region, the plane below the TPF is lost, $3 \mathrm{~cm}$ behind the lateral border of the orbit to a point along the lateral orbital border that is $2.3 \mathrm{~cm}$ superior to the zygomatic arch. ${ }^{15}$ According to their study there is no loose areolar tissue, the fascia is a thick fibro-fatty membrane, united with the STF via multiple adhesions. This adhesion between the TPF and temporalis fascia is also described by others as the inferior temporal septum, lying an average $27 \mathrm{~mm}$ above the zygoma at the level of the frontal process of the zygoma and $21 \mathrm{~mm}$ above the superior border of the zygoma at its midportion. ${ }^{17}$ Due to the fusion of these three layers (TPF, loose areolar tissue, STF), there is no clear dissection plane, and the FTB of the facial nerve is at a greater risk of injury in case a dissection is performed to reach the zygomatic arch.

In contrast, over the superolateral angle of the orbital rim, the FTB courses above the TPF. The loose areolar tissue between the TPF and the STF is present and easily dissectible. ${ }^{15,18,19}$ There are no adhesions between the two fascial layers in this location, and the dissection can be easily and safely performed. Although we did not perform histologic sections in our study, during the microsurgical dissection of the facial nerve, the thick adhesions between the individual fascial layers were also identified in the anterior zygomaticotemporal region (- Fig. $\mathbf{1}$ ).

Therefore if interfascial dissection is contemplated, it can be performed safely until the fibrous attachment between the TPF, loose areolar tissue, and temporal fascia are reached. ${ }^{17,19}$ Once the landmark where the adhesions begin is reached, it is safer to keep the dissection plane in the loose areolar tissue between the TPF and the temporalis fascia close to the superior temporal line where this dissection plane between the two layers is apparent and safe for the FTB of the facial nerve and not below the frontozygomatic suture, anteriorly in the zygomaticotemporal region. By making a cut on the STF on the superior temporal line (where the STF and DTF are continuous), one can easily identify both fasciae and the interfascial fat pad. The oblique cut of the STF toward the root of the zygoma avoids the anteriorly located risk zone for the FTB. ${ }^{15,19}$ By elevating the SFT with the scissors tip by each cut, one can avoid mistaking the individual tissue planes.

The STF in its most anterior part is very thin and transparent, and the interfascial (between the STF and the DTF) fat pad could sometimes be visualized (-Fig. 1). As in the first description by Yaşargil et al, ${ }^{16}$ the STF cut should be done when the interfascial fat pad is noted. However, as Ammirati et $\mathrm{al}^{11}$ point out in their paper, one could mistake the subgaleal fat pad for the interfascial one, which increases the chances for mistakes and injury to the FTB as one may slip from the STF into the subgaleal plane during interfascial dissection. By starting the dissection from the superior temporal line as previously described, one could possibly avoid such a mistake. As long as the following dissection is below the interfascial fat pad, the risk for the FTB is minimal $2,13,15,16,26$ (- Fig. 1).

At the level of the zygomatic arch, a subperiosteal dissection is the key to protect the facial nerve and should start either from the orbital rim down and backward or from the level above the temporomandibular joint forward. ${ }^{19}$

As an alternative to the interfascial dissection, the combined frontotemporal scalp/temporal fascia dissection (subfascial dissection) could be performed that is anatomically suited to preserve the temporal branch of the facial nerve and its variations that may run within the fat pad. ${ }^{10,12,14,15}$ The DTF is incised on the medial aspect of the zygoma to expose it. With this maneuver, both fascial layers are released, and the flap can be reflected forward and over the lateral side of the zygoma. There is virtually no chance to injure the FTB of the facial nerve ${ }^{14}$ (-Figs. 2 and 4 ).

\section{Conclusion}

A clear understanding of the complex relationship between the individual tissue planes and the course of the FTB of the facial nerve are needed to perform $\mathrm{OZ}$ approaches safely. If an interfascial approach is contemplated, it is safe to start the dissection from the most anterior aspect of the superior temporal line and to cut the STF obliquely toward the root of the zygoma and not extend the dissection in the anterior zygomaticotemporal area below the frontozygomatic suture because of the danger of injury to the FTB of the facial nerve. 
Further, the dissection should be kept close to the DTF below the fat pad. However, the subfacial dissection offers a simple and safe elevation of both temporalis fasciae with the interfascial fad pad, and thus it protects the FTB including its potential variable twig.

\section{Notes}

We presented this work as an e-poster at the World Federation of Neurosurgical Societies (WFNS) meeting, Seoul, South Korea, September 8-13, 2013.

\section{References}

1 al-Mefty O, Anand VK. Zygomatic approach to skull-base lesions. J Neurosurg 1990;73(5):668-673

2 Aziz KM, Froelich SC, Cohen PL, Sanan A, Keller JT, van Loveren HR. The one-piece orbitozygomatic approach: the MacCarty burr hole and the inferior orbital fissure as keys to technique and application. Acta Neurochir (Wien) 2002;144(1):15-24

3 Cheng CM, Chang CF, Ma HI, Chiang YH, McMenomey SO, Delashaw JB Jr. Modified orbitozygomatic craniotomy for large medial sphenoid wing meningiomas. J Clin Neurosci 2009; 16(9):1157-1160

4 Deshmukh VR, Albuquerque FC, Zabramski JM, Spetzler RF. Surgical management of cavernous malformations involving the cranial nerves. Neurosurgery 2003;53(2):352-357; discussion 357

5 Golshani KJ, Lalwani K, Delashaw JB, Selden NR. Modified orbitozygomatic craniotomy for craniopharyngioma resection in children. J Neurosurg Pediatr 2009;4(4):345-352

6 Gonzalez LF, Crawford NR, Horgan MA, Deshmukh P, Zabramski JM, Spetzler RF. Working area and angle of attack in three cranial base approaches: pterional, orbitozygomatic, and maxillary extension of the orbitozygomatic approach. Neurosurgery 2002; 50(3):550-555; discussion 555-557

7 Liu JK, Fukushima T, Sameshima T, Al-Mefty O, Couldwell WT. Increasing exposure of the petrous internal carotid artery for revascularization using the transzygomatic extended middle fossa approach: a cadaveric morphometric study. Neurosurgery 2006; 59(4, Suppl 2):ONS309-ONS318; discussion ONS318-ONS319

8 Sindou M, Emery E, Acevedo G, Ben-David U. Respective indications for orbital rim, zygomatic arch and orbito-zygomatic osteotomies in the surgical approach to central skull base lesions. Critical, retrospective review in 146 cases. Acta Neurochir (Wien) 2001;143(10):967-975

9 Shimizu S, Tanriover N, Rhoton AL Jr, Yoshioka N, Fujii K. MacCarty keyhole and inferior orbital fissure in orbitozygomatic craniotomy. Neurosurgery 2005;57(1, Suppl):152-159; discussion 152-159

10 Zabramski JM, Kiriş T, Sankhla SK, Cabiol J, Spetzler RF. Orbitozygomatic craniotomy. Technical note. J Neurosurg 1998;89(2): 336-341
11 Ammirati M, Spallone A, Ma J, Cheatham M, Becker D. An anatomicosurgical study of the temporal branch of the facial nerve. Neurosurgery 1993;33(6):1038-1043; discussion 1044

12 Ammirati M, Spallone A, Ma J, Cheatham M, Becker D. Preservation of the temporal branch of the facial nerve in pterional-transzygomatic craniotomy. Acta Neurochir (Wien) 1994;128(1-4): 163-165

13 Aoki N. Incision of facial nerve branch at aneurysm surgery [letter]. J Neurosurg 1987;66(3):482

14 Coscarella E, Vishteh AG, Spetzler RF, Seoane E, Zabramski JM. Subfascial and submuscular methods of temporal muscle dissection and their relationship to the frontal branch of the facial nerve. Technical note. J Neurosurg 2000;92(5):877-880

15 Salas E, Ziyal IM, Bejjani GK, Sekhar LN. Anatomy of the frontotemporal branch of the facial nerve and indications for interfascial dissection. Neurosurgery 1998;43(3):563-568; discussion 568-569

16 Yaşargil MG, Reichman MV, Kubik S. Preservation of the frontotemporal branch of the facial nerve using the interfascial temporalis flap for pterional craniotomy. Technical article. J Neurosurg 1987;67(3):463-466

17 Moss CJ, Mendelson BC, Taylor GI. Surgical anatomy of the ligamentous attachments in the temple and periorbital regions. Plast Reconstr Surg 2000;105(4):1475-1490; discussion 1491-1498

18 Davidge KM, van Furth WR, Agur A, Cusimano M. Naming the soft tissue layers of the temporoparietal region: unifying anatomic terminology across surgical disciplines. Neurosurgery 2010; 67(3, Suppl Operative):ons120-ons129; discussion ons129-ons130

19 Krayenbühl N, Isolan GR, Hafez A, Yaşargil MG. The relationship of the fronto-temporal branches of the facial nerve to the fascias of the temporal region: a literature review applied to practical anatomical dissection. Neurosurg Rev 2007;30(1):8-15; discussion 15

20 Lei T, Xu DC, Gao JH, et al. Using the frontal branch of the superficial temporal artery as a landmark for locating the course of the temporal branch of the facial nerve during rhytidectomy: an anatomical study. Plast Reconstr Surg 2005;116(2):623-629; discussion 630

21 Abul-Hassan HS, von Drasek Ascher G, Acland RD. Surgical anatomy and blood supply of the fascial layers of the temporal region. Plast Reconstr Surg 1986;77(1):17-28

22 Campiglio GL, Candiani P. Anatomical study on the temporal fascial layers and their relationships with the facial nerve. Aesthetic Plast Surg 1997;21(2):69-74

23 Sabini P, Wayne I, Quatela VC. Anatomical guides to precisely localize the frontal branch of the facial nerve. Arch Facial Plast Surg 2003;5(2):150-152

24 Stuzin JM, Wagstrom L, Kawamoto HK, Wolfe SA. Anatomy of the frontal branch of the facial nerve: the significance of the temporal fat pad. Plast Reconstr Surg 1989;83(2):265-271

25 Gosain AK, Sewall SR, Yousif NJ. The temporal branch of the facial nerve: how reliably can we predict its path? Plast Reconstr Surg 1997;99(5):1224-1233; discussion 1234-1236

26 Hwang K, Suh MS, Lee SI, Chung IH. Zygomaticotemporal nerve passage in the orbit and temporal area. J Craniofac Surg 2004; 15(2):209-214 